\title{
Overcoming the Efficiency Barrier of Textile Antennas: A Transmission Lines Approach ${ }^{\dagger}$
}

\author{
Mahmoud Wagih *, Alex S. Weddell and Steve Beeby \\ School of Electronics and Computer Science, University of Southampton, Southampton SO17 1BJ, UK; \\ asw@ecs.soton.ac.uk (A.S.W.); spb@ecs.soton.ac.uk (S.B.) \\ * Correspondence: mahm1g15@ecs.soton.ac.uk; Tel.: +44-2380-593-234 \\ + Presented at the International Conference on the Challenges, Opportunities, Innovations and Applications \\ in Electronic Textiles (E-Textiles 2019), London, UK, 12 November 2019.
}

Published: 11 December 2019

\begin{abstract}
Designing high-efficiency antennas on textiles is fundamental for the development of wirelessly-connected smart garments. Furthermore, large antenna arrays could be used to receive or harvest directional and ambient radio-frequency (RF) power from the environment, thus enabling battery-free e-textiles. The key challenges that are hindering the realisation of high efficiency antennas lie in the dielectric properties of fabrics, the conductivity of their traces, and their low textile thickness. This work numerically and experimentally analyses different RF transmission line structures to establish the limitations of widely utilised antenna designs, such as the microstrip patch, and proposes alternative wearable antenna design based on coplanar waveguide (CPW) structures. It is demonstrated that by using a CPW, insertion losses in a $20 \mathrm{~mm}$ line can be minimized by up to $40 \%$ for the same substrate, as compared to a microstrip, at $30 \mathrm{GHz}$. A CPW monopole antenna is demonstrated with more than $80 \%$ efficiency on a lossy, thin, poly-cotton substrate. Moreover, it is shown that the efficiency of the CPW monopole is independent of the substrate's thickness and type of fabric.
\end{abstract}

Keywords: antenna; body area networks; coplanar waveguide; e-textiles; microstrip

\section{Introduction}

Smart e-textiles are a promising platform for body area wireless sensor networks. Antenna design on textiles has been an increasingly popular research area with fully-textile, hybrid and fullyrigid antennas designed for integration in garments [1]. Flexible and textile antennas have been previously presented for body area networks [2,3], for energy harvesting at $2.4 \mathrm{GHz}$ [4], and at 5G millimetre-wave (mmWave) bands [5]. Furthermore, substrate integrated waveguides (SIW) have been fabricated on textiles by using different conductive materials for improved isolation [1].

Flexible on-body antenna design has been mostly focused on microstrip patch antennas and microstrip transmission lines [3,4], two areas which require geometrical limitations on the textile substrate, such as a large thickness, or bottlenecks the antenna's radiation efficiency and bandwidth. For example, the radiation efficiency of a polyester-cotton patch antenna fabricated using inkjet printing has been capped at $56 \%$ [3]. Furthermore, to achieve a radiation efficiency of $73 \%$ in [4], the microstrip patch antenna had to be designed on a multi-layer felt substrate of more than $3 \mathrm{~mm}$ thickness utilising metalized conductive fabric for the antenna minimizing both the dielectric and conductor losses. At $5 \mathrm{G}$ mmWave bands (24-30 GHz), a radiation efficiency of $77.5 \%$ was reported in [5] by using an antipodal Vivaldi monopole fabricated on a relatively thin woven-polyester substrate through the use of etched flexible copper laminates for the antenna's conductive traces. 
To overcome the efficiency barrier of textile antennas through an alternative design to the commonly used patch, this paper reviews the suitability of common e-textile fabrication techniques for radio-frequency (RF) networks and antennas. The experimental characterisation of a standard textile substrate up to $67 \mathrm{GHz}$ is presented and the insertion losses in different textile transmission line structures are analysed. A coplanar wave guide (CPW) monopole antenna is designed based on the transmission line analysis and demonstrates a higher radiation efficiency than state-of-art textile microstrip antennas.

\section{Characterising and Analysing Textile Transmission Lines}

The losses in a RF transmission line, and hence in an antenna, are categorized into conductor and dielectric losses. The conductor losses are a main function of the conductor's skin depth and surface roughness [6], and the dielectric losses are usually a function of the antenna's geometry [5].

Multiple e-textile fabrication techniques are based on additive manufacturing: Conductive ink is deposited on a printed interface layer which reduces the roughness of the textile to around $10 \mu \mathrm{m}$ [2]. While this technique is suitable for Direct Current (DC) and low frequency applications, when the skin depth effect is combined with the $10 \mu \mathrm{m}$ surface roughness in [3], the conductive losses result in a reduced radiation efficiency. Moreover, the commercially available interface layers characterized in [4] suffer from a high dissipation factor $(\tan \delta)$, which increases the dielectric losses of the line or the antenna.

An alternative fabrication technique is the utilisation of thin copper laminates [3,5] or metallised conductive textiles [4]. By using polyimide copper laminates or metallised fabrics, the surface roughness effect will be minimised to commercial printed circuit board-levels, thus reducing the conductor losses compared to thin-films techniques. Moreover, this eliminates the need for printed interface layers of high dissipation factors, an interface which reduces the radiation efficiency of planar antennae.

To accurately model the properties of textile lines, the fabric substrate was experimentally characterized by using the two-line dielectric characterisation technique [7]. The two-port scattering matrices of two microstrip lines of different lengths were measured using a vector network analyser (VNA) to extract the dielectric properties of the textile [7]. The extracted dielectric properties of the woven-polyester with polyimide interface layer were $\varepsilon_{r}=1.67$ and $\tan \delta=0.028$. Figure 1 shows the measured scattering-parameters results as well as the measurement technique

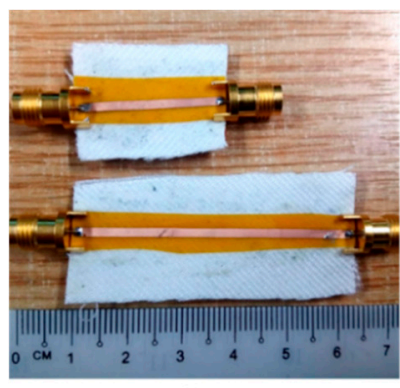

(a)

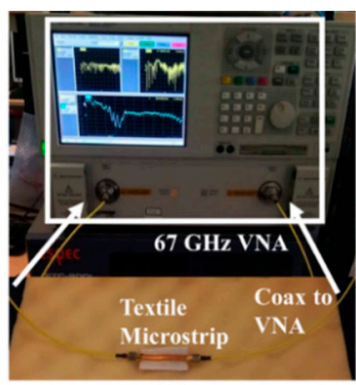

(b)

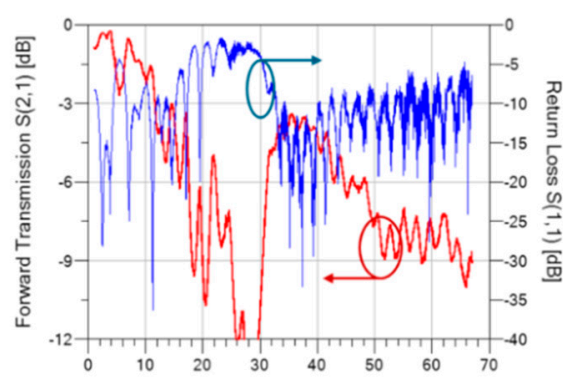

(c)

Figure 1. Textile microstrip substrate characterization: (a) 55 and $20 \mathrm{~mm}$ woven-polyester microstrips; (b) two-port measurement setup using a vector network analyser (VNA) up to $67 \mathrm{GHz}$; and (c) measured s-parameters of the microstrip lines.

Based on the extracted parameters of the textile substrate, a microstrip and a CPW were built in 3D full-wave electromagnetic simulation in CST Microwave Studio, a finite integration technique (FIT) package. The simulated transmission lines were designed based on a $200 \mu \mathrm{m}$ woven-polyester substrate, with a $100 \mu \mathrm{m}$ printed waterproof interface layer $\left(\varepsilon_{\mathrm{r}}=1.67\right.$ and $\left.\tan \delta=0.028[4]\right)$ as in $[2,4]$. Both lines were designed to have a $50 \Omega$ characteristic impedance and a length of $20 \mathrm{~mm}$. Figure 2 shows the electric field distribution of the textile CPW and microstrip lines. 
(a)
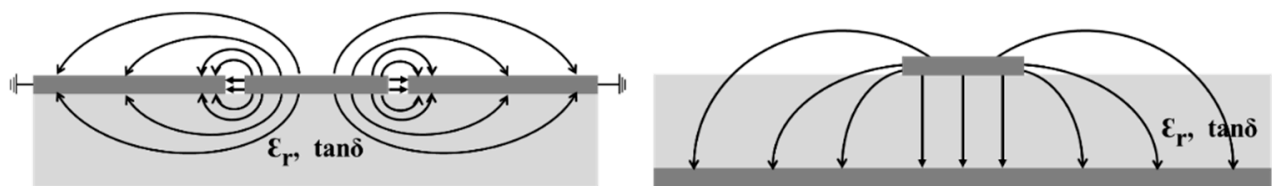

(b)

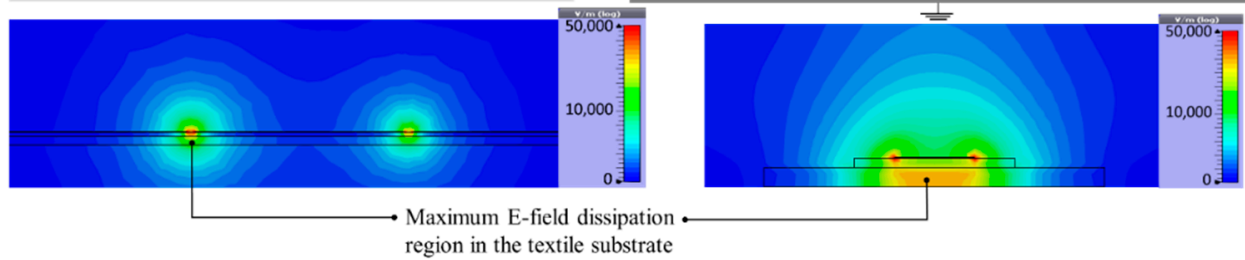

Figure 2. Electric-field lines (a) and simulated electric field patterns (b) in the coplanar wave guide (CPW) (left) and microstrip (right) transmission lines at $5 \mathrm{GHz}$.

Due to the electric field distribution in Figure 2, the insertion losses on a lossy dielectric were expected to be higher for a microstrip line due to the higher electric-field dissipation in the substrate. Figure 3 shows the forward transmission of the $20 \mathrm{~mm}$ lines, up to $50 \mathrm{GHz}$, of the $10 \mu \mathrm{m}$ conductor surface-roughness, with more than $37 \%$ higher losses in the microstrip line compared to the CPW. Moreover, the CPW line could be used up to $10 \mathrm{GHz}$ higher than a microstrip line of similar losses, thus demonstrating its suitability for mmWave textile antennas and filters for future $5 \mathrm{G}$ networks.
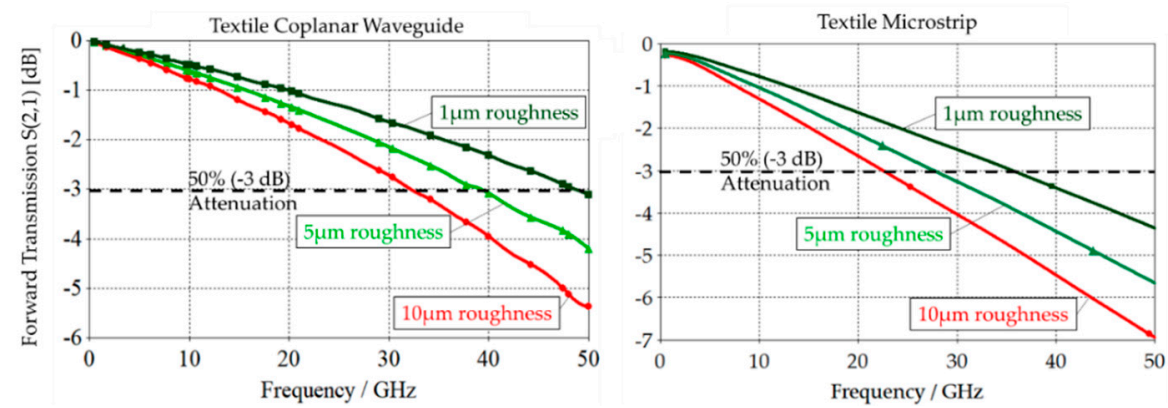

Figure 3. Forward transmission ( $\left.\mathrm{S}_{21}\right)$ of the textile $\mathrm{CPW}$ and microstrip line showing lower losses in the CPW.

\section{The Coplanar-Wave Guide Monopole Antenna}

As a CPW line was demonstrated to reduce insertion losses, a quarter-wave monopole antenna fed using a $50 \mathrm{Ohm} \mathrm{CPW}$ was designed and simulated for a textile substrate for the $2.4 \mathrm{GHz}$ Industrial, Scientific Medical (ISM)-band. The antenna was fabricated using polyimide copper laminates, which were plotter-cut to create the trace pattern, and adhered to a thin $(<0.3 \mathrm{~mm})$ polyester-cotton substrate using 3M spray-mount adhesive. Figure 4 shows the antenna's layout and electric field distribution, as well as the antenna's photograph on-body.

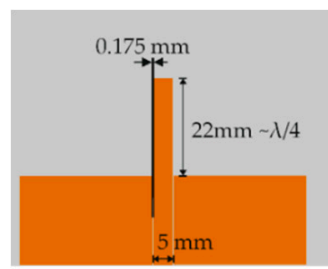

(a)

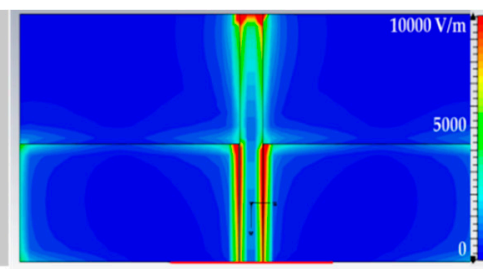

(b)

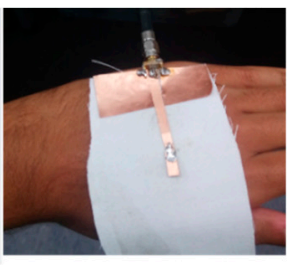

(c)

Figure 4. The CPW textile 2.4 GHz monopole antenna. (a): layout; (b): simulated E-field pattern, and (c): fabricated antenna on-hand.

The return loss of the antenna was measured using a VNA with a soldered SMA connector (Figure 4c) to validate the simulated results. Figure 5 shows the simulated and measured return loss, which showed a close agreement around $2.4 \mathrm{GHz}$, as well as the computed 3D gain radiation pattern. 

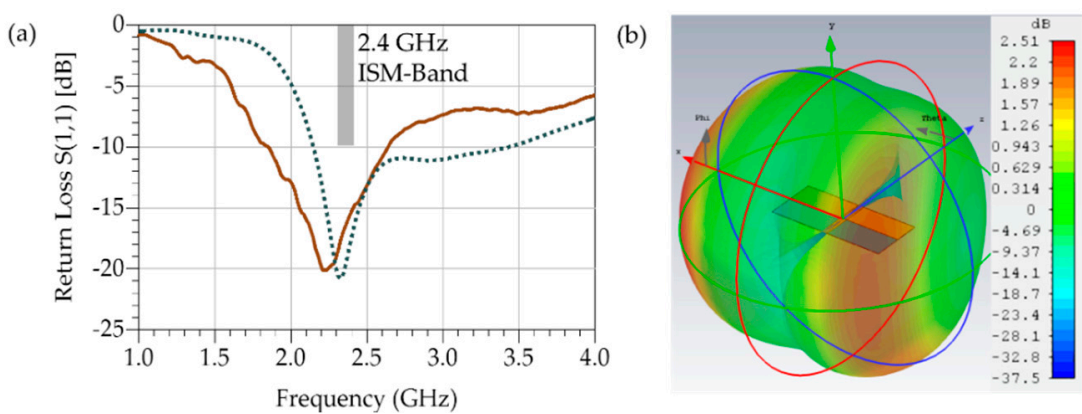

Figure 5. Performance of the CPW textile monopole: (a) Simulated (dashed) and measured (solid) return loss showing broadband operation around $2.4 \mathrm{GHz}$ and (b) 3D radiation patterns at $2.4 \mathrm{GHz}$ and $2.51 \mathrm{~dB}$ gain.

As predicted based on the transmission line analysis, the antenna a demonstrated low radiation resistance (conductor and dielectric losses) resulting in a higher radiation efficiency compared to patch antennas due to the lower losses of a CPW monopole antenna. Table 1 shows the simulated and measured antenna parameters as well as a comparison with printed and metalized-fabric patch antennas on thick felt fabrics. The proposed antenna's gain was lower than the reported antennas in [2] and [4] due to the omnidirectional radiation pattern of the proposed antenna. A $50 \%$ higher radiation efficiency could translate to a lower transmission power while maintaining the same range for a textile transmitter or to $50 \%$ more received power in case of a wireless power receiver.

Table 1. Antenna parameters and comparison with state-of-art textile microstrip antennas.

\begin{tabular}{cccc}
\hline & This Work & Whittow et al. (2014) [2] & Adami et al. (2018) [4] \\
\hline Antenna Design & CPW Monopole & Microstrip Patch & Microstrip Patch \\
\hline Conductor & Cu Polyimide Laminate & Inkjet-Printed Silver Ink & Copper-Coated Fabric \\
\hline $\begin{array}{c}\text { Substrate } \\
\text { (tan } \delta=0.028)\end{array}$ & $\begin{array}{c}0.2 \mathrm{~mm} \text { Woven-Polyester }+ \\
1.4 \mathrm{~mm} \text { Felt (tan } \delta=0.023)\end{array}$ & $\begin{array}{c}3.2 \mathrm{~mm} \text { Felt } \\
(\tan \delta=0.028)\end{array}$ \\
\hline $\begin{array}{c}\text { Return Loss, } \mathbf{S}_{11} \\
\text { (dB) }\end{array}$ & -20.1 & -9 & -26 \\
\hline $\begin{array}{c}\text { Fractional } \\
\text { Bandwidth }\end{array}$ & $35 \%$ & $0.58 \%$ & $4.1 \%$ \\
\hline $\begin{array}{c}\text { Radiation } \\
\text { Efficiency }\end{array}$ & $86 \% *$ & $37 \%$ & $73 \%$ \\
\hline \begin{tabular}{c} 
Gain (dB) \\
\hline
\end{tabular} & $2.51 *$ & 4.02 & 8.1 \\
\hline
\end{tabular}

* Simulated using material-accurate model based on empirical characterization in Section 2.

\section{Conclusions}

This paper has presented experimental and numerical analysis of the impact of e-textile fabrication techniques on insertion losses. Transmission lines were analysed as a method of characterising the losses associated with RF textiles by devising a high-efficiency antenna design technique. A high efficiency textile antenna has been presented based on a CPW structure, and this antenna demonstrates significant performance improvements over state-of-art microstrip textile antennae. These improvements were achieved by reducing the dielectric and conductor losses and reducing the dependence on the textile thickness and dielectric properties. This advance in textile antenna efficiency enables more power-efficient body area networks and efficient RF energy harvesting on textiles.

Author Contributions: Conceptualization: M.W.; Methodology: M.W.; Writing-Original Draft Preparation: M.W.; Writing-Review \& Editing: M.W., A.S.W. and S.B.; Supervision: A.S.W. and S.B.

Funding: This work was supported by the European Commission under H2020-EU.1.4.1.2.

Conflicts of Interest: The authors declare no conflict of interest. 


\section{References}

1. Corchia, L.; Monti, G.; Tarricone, L. Wearable Antennas: Nontextile Versus Fully Textile Solutions. IEEE Antennas Propag. Mag. 2019, 61, 71-83.

2. Whittow, W.G.; Chauraya, A.; Vardaxoglou, J.C.; Li, Y.; Torah, R.; Yang, K.; Beeby, S.; Tudor, J. InkjetPrinted Microstrip Patch Antennas Realized on Textile for Wearable Applications. IEEE Antennas Wirel. Propag. Lett. 2014, 13, 71-74.

3. Wagih, M.; Wei, Y.; Beeby, S. Flexible 2.4 GHz Node for Body Area Networks with a Compact High-Gain Planar Antenna. IEEE Antennas Wirel. Propag. Lett. 2018, 18, 49-53.

4. Adami, S.E.; Proynov, P., Hilton, G. S., Yang, G., Zhang, C., Zhu, D.; Li, Y.; Beeby, S.P.; Craddock, I.J.; Stark, B.H. A Flexible 2.45-GHz Power Harvesting Wristband with Net System Output from $-24.3 \mathrm{dBm}$ of RF Power. IEEE Trans. Microw. Theory Tech. 2017, 66, 380-395.

5. Wagih, M.; Weddell, A.S.; Beeby, S. Millimeter-Wave Textile Antenna for On-Body RF Energy Harvesting in Future 5G Networks. In Proceedings of the IEEE Wireless Power Transfer Conference, London, UK, 1821 June 2019.

6. Rotaru, M.D.; Wagih, M.A. Analysis and Design of Low-Loss Differential Transmission Line Structures for High Speed Applications. In Proceedings of the IEEE 19th Electronic Packaging Technology Conference, Singapore, 6-9 December 2017.

7. Declercq, F.; Rogier, H.; Hertleer, C. Permittivity and Loss Tangent Characterization for Garment Antennas Based on a New Matrix-Pencil Two-Line Method. IEEE Trans. Antennas Propag. 2008, 56, 2548-2554.

(C) 2019 by the authors. Licensee MDPI, Basel, Switzerland. This article is an open access article distributed under the terms and conditions of the Creative Commons Attribution (CC BY) license (http://creativecommons.org/licenses/by/4.0/). 